\title{
Inclusive production of a Higgs or $Z$ boson in association with heavy quarks
}

\author{
Fabio Maltoni \\ Theoretical Physics Division, CERN, 1211-CH Geneva, Switzerland \\ Thomas McElmurry and Scott Willenbrock \\ Department of Physics, University of Illinois at Urbana-Champaign, 1110 West Green Street, Urbana, Illinois 61801, USA
}

(Received 23 May 2005; published 21 October 2005)

\begin{abstract}
We calculate the cross section for the production of a $Z$ boson in association with heavy quarks. We suggest that this cross section can be measured using an inclusive heavy-quark tagging technique. This could be used as a feasibility study for the search for a Higgs boson produced in association with bottom quarks. We argue that the best formalism for calculating that cross section is based on the leading-order process $b \bar{b} \rightarrow h$, and that it is valid for all Higgs masses of interest at both the Fermilab Tevatron and the CERN Large Hadron Collider.
\end{abstract}

DOI: 10.1103/PhysRevD.72.074024

PACS numbers: 13.85.Qk, 12.38.Bx, 14.70.Hp

\section{INTRODUCTION}

In the standard model, the Higgs boson has a very weak coupling to bottom quarks. However, in a two-Higgsdoublet model, the coupling of some or all of the physical Higgs particles to bottom quarks can be greatly enhanced. For example, this occurs in the minimal supersymmetric model for large values of $\tan \beta \equiv v_{2} / v_{1}$, where $v_{1}$ and $v_{2}$ are the vacuum expectation values of the Higgs doublets that couple to bottom and top quarks, respectively. If the coupling is sufficiently enhanced, the production of Higgs bosons in association with bottom quarks can be an important process at the Fermilab Tevatron $(p \bar{p}, \sqrt{S}=$ $1.96 \mathrm{TeV}$ ) and the CERN Large Hadron Collider (LHC) $(p p, \sqrt{S}=14 \mathrm{TeV})$. A great deal of attention has been directed towards this process [1-26].

In order to separate the signal from the background, and also to identify the production process, it is advantageous to tag one or more of the bottom quarks produced along with the Higgs boson (in addition to the bottom quarks that might result from Higgs decay). Up until now, this has been discussed as the identification of a high- $p_{T} b$-tagged jet. However, there exist more inclusive means to identify bottom quarks in the final state at hadron colliders, such as identifying a secondary vertex without requiring the reconstruction of a high- $p_{T}$ jet [27]. In this paper we would like to lay the groundwork for such a measurement.

As a testing ground for the Higgs, we propose a measurement of the inclusive production of a $Z$ boson in association with heavy quarks. ${ }^{1}$ This is more complicated than the Higgs case for three reasons. First, the $Z$ boson is produced in association with both bottom and charm quarks, so both possibilities must be taken into account. Second, $Z$ bosons are dominantly produced in association with light quarks, which can fake a heavy quark. Third, the processes $q \bar{q} \rightarrow Z Q \bar{Q}$ and $q Q \rightarrow Z q Q(Q=c, b)$, where

\footnotetext{
${ }^{1}$ The production of a $Z$ boson in association with a heavyquark jet is dealt with in Refs. [28,29].
}

the $Z$ couples to the light quarks, are contributions that have no analogue in the Higgs case.

There is a second motivation for this paper. There exist two different formalisms for the calculation of inclusive Higgs production in association with bottom quarks. The first is based on the leading-order (LO) process $g g \rightarrow h b \bar{b}$, the second on the LO process $b \bar{b} \rightarrow h .^{2}$ The advantage of the latter formalism is that it resums, to all orders in perturbation theory, collinear logarithms of the form $\ln \left(m_{h} / m_{b}\right)$ that arise in the calculation based on $g g \rightarrow$ $h b \bar{b}$ [30,31]. It has recently been suggested that both formalisms may be unreliable for Higgs bosons at the Tevatron [32]. We will show evidence that the calculation based on $b \bar{b} \rightarrow h$ is reliable for Higgs masses of experimental interest, and argue for its superiority. However, we also find evidence that the formalism fails as the Higgs mass approaches the machine energy, in agreement with Ref. [32].

The paper is organized as follows. We first discuss, in Sec. II, the calculation of $b \bar{b} \rightarrow h$, and argue that it is reliable for all Higgs masses of interest at the Tevatron and LHC. We then turn in Sec. III to inclusive production of a $Z$ boson with heavy quarks. Readers who are only interested in the latter topic may skip directly to that section. We conclude with a discussion of our results.

\section{HIGGS PRODUCTION IN ASSOCIATION WITH HEAVY QUARKS}

Inclusive Higgs production in association with bottom quarks may be calculated in two different schemes. One may work in a four-flavor scheme, where the LO process is $g g \rightarrow h b \bar{b}$. This approach yields collinear logarithms of the form $\ln \left(m_{h} / m_{b}\right)$, which degrade the convergence of the perturbation series. Alternatively, one may work in a fiveflavor scheme, where the LO process is $b \bar{b} \rightarrow h[30,31]$.

\footnotetext{
${ }^{2}$ When a $b$ distribution function is used, it is implicit that there is a spectator $\bar{b}$ in the final state.
} 

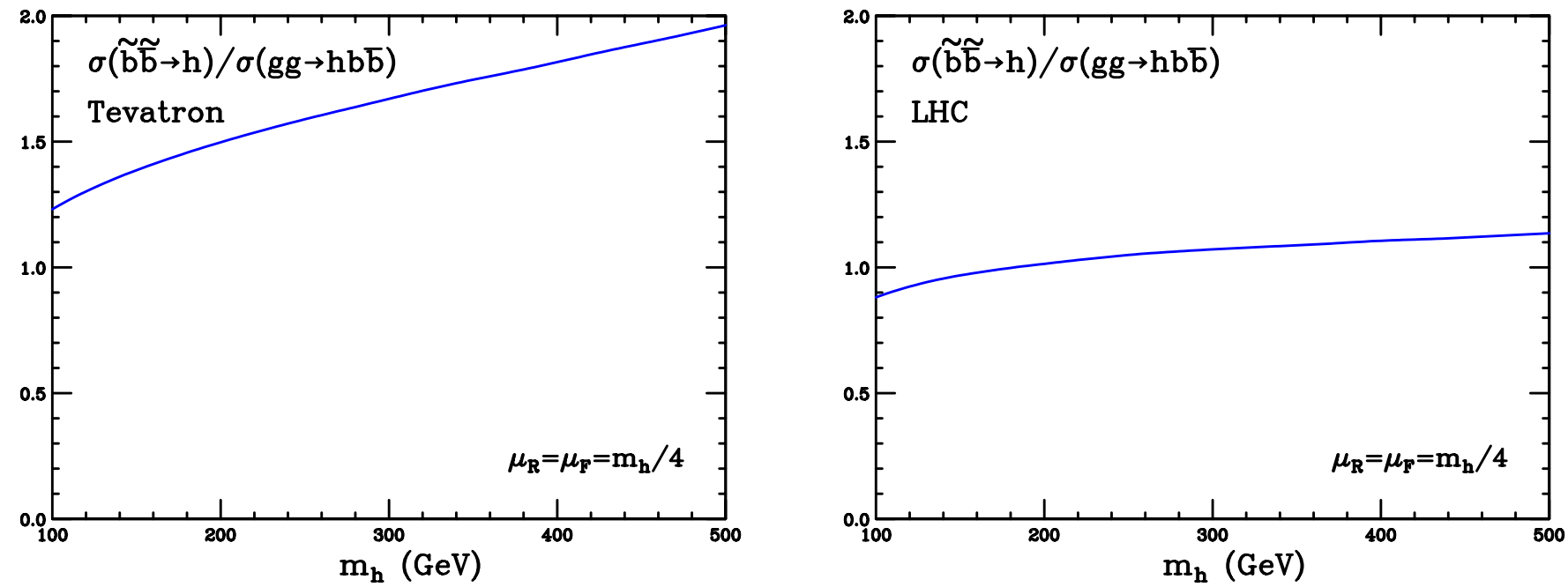

FIG. 1 (color online). $\quad \sigma(\tilde{b} \tilde{\tilde{b}} \rightarrow h) / \sigma(g g \rightarrow h b \bar{b})$ vs $m_{h}$ at the Tevatron and the LHC, using MRST2001 LO parton distribution functions [43], $m_{b}=4.7 \mathrm{GeV}$, and $\mu_{F}\left(=\mu_{R}\right)=m_{h} / 4$.

The calculation based on $b \bar{b} \rightarrow h$ yields a more convergent perturbation series, since the collinear logarithms are summed into the $b$-quark distribution functions via the Dokshitzer-Gribov-Lipatov-Altarelli-Parisi (DGLAP) equations. As one calculates to higher and higher order in perturbation theory, the two calculations should approach each other, since they are simply different orderings of the same terms.

The collinear logarithms that arise in $g g \rightarrow h b \bar{b}$ at LO can be captured by an approximate $b$-quark distribution function,

$$
\tilde{b}\left(x, \mu_{F}\right)=\frac{\alpha_{S}\left(\mu_{F}\right)}{2 \pi} \ln \left(\frac{\mu_{F}^{2}}{m_{b}^{2}}\right) \int_{x}^{1} \frac{d y}{y} P_{q g}\left(\frac{x}{y}\right) g\left(y, \mu_{F}\right)
$$

where $P_{q g}(x)=\frac{1}{2}\left[x^{2}+(1-x)^{2}\right]$ is the LO DGLAP splitting function and $\mu_{F}$ is the factorization scale, of order $m_{h}$. Unlike the exact $b$ distribution function, the approximate $b$ distribution function does not sum the collinear logarithms. Thus the calculations $g g \rightarrow h b \bar{b}$ and $\tilde{b} \bar{b} \rightarrow h$ should approximately agree if the terms enhanced by collinear logarithms in $g g \rightarrow h b \bar{b}$ are dominant.

Recently it was noted that the calculations $g g \rightarrow h b \bar{b}$ and $\tilde{b} \tilde{b} \rightarrow h$ differ substantially at LO for heavy Higgs bosons $\left(m_{h}>100 \mathrm{GeV}\right)$ at the Tevatron, the discrepancy increasing with increasing Higgs mass [32]. In contrast, the two calculations agree fairly well at the LHC for $m_{h}=$ $100-500 \mathrm{GeV}$ as well as at the Tevatron for $m_{h}<$ $100 \mathrm{GeV}$. Reference [32] concludes that both calculations are suspect at the Tevatron for $m_{h}>100 \mathrm{GeV}$.

We show in Fig. 1 the ratio of $\tilde{b} \bar{b} \rightarrow h$ to $g g \rightarrow h b \bar{b}$ at both the Tevatron and the LHC. These results agree closely with those of Ref. [32]. We see that the ratio is about 1.5 for $m_{h}=200 \mathrm{GeV}$ at the Tevatron, increasing to nearly 2 for $m_{h}=500 \mathrm{GeV}$.
Implicit in this argument is the choice of the factorization scale. It was argued in Ref. [20] that the appropriate factorization scale is $\mu_{F} \approx m_{h} / 4$, and this is the scale that was used in Ref. [32] and Fig. 1. We show below that for heavy Higgs bosons at the Tevatron, a slightly lower scale is more appropriate, and that this partially resolves the large discrepancy between the LO calculations of $g g \rightarrow$ $h b \bar{b}$ and $\tilde{b} \tilde{b} \rightarrow h$.

The argument for the factorization scale made in Ref. [20] is based on an analysis of the collinear logarithm that arises at next-to-leading order (NLO) in the calculation of $b \bar{b} \rightarrow h$, and is in the same spirit as the argument of Refs. $[33,34]$. In the collinear region, the NLO differential hadronic cross section scales like $d \sigma / d t \sim 1 / t$, where $t$ is the usual Mandelstam variable. We show in Fig. 2 the quantity $-t d \sigma / d t$ vs $\sqrt{-t} / m_{h}$ for the NLO process $g b \rightarrow h b$ at both the Tevatron and the LHC for $m_{h}=100-500 \mathrm{GeV}^{3}$ The factorization scale should be chosen near the end of the collinear plateau. At the LHC this plateau ends around $m_{h} / 4$ for the Higgs-boson masses considered. However, at the Tevatron the end of the plateau slowly creeps below $m_{h} / 4$ as the Higgs-boson mass increases (this is also true at the LHC, but much less so).

To be consistent, we choose the factorization scale to be where $-t d \sigma / d t$ reaches $85 \%$ of its value on the collinear plateau. The resulting factorization scale at both the Tevatron and the LHC is given in Table I. We show in Fig. 3 the ratio of $\tilde{b} \overline{\tilde{b}} \rightarrow h$ to $g g \rightarrow h b \bar{b}$ at both the Tevatron and the LHC with this choice of factorization scale. The ratio approaches unity for large Higgs masses at

\footnotetext{
${ }^{3}$ Since this is a NLO process, we use NLO parton distribution functions [35]. We use $\mu_{F}=m_{h}$ (the default value), as this graph is being used to determine $\mu_{F}$. We subsequently check that the curves are not very sensitive to the choice of $\mu_{F}$.
} 

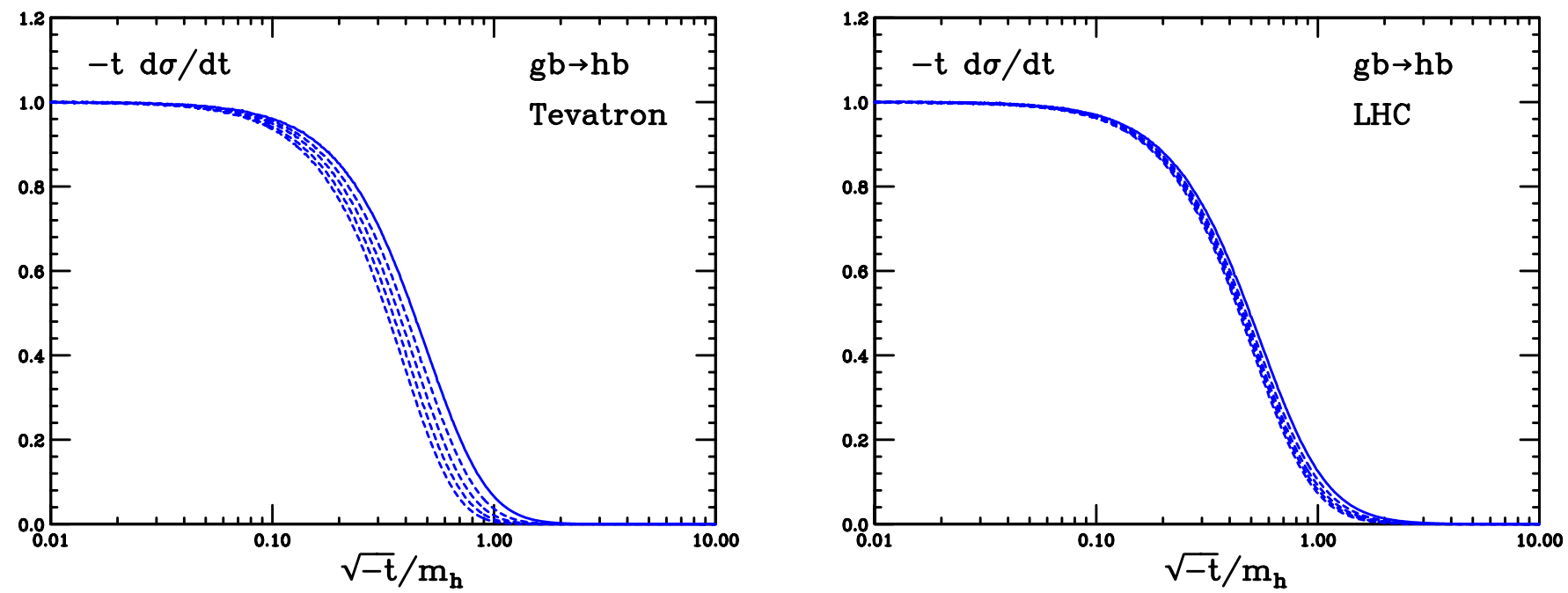

FIG. 2 (color online). $\quad-t d \sigma / d t$ vs $\sqrt{-t} / m_{h}$ for $g b \rightarrow h b$ at the Tevatron and the LHC. The factorization scale for $b \bar{b} \rightarrow h$ should be chosen near the end of the collinear plateau.

TABLE I. The factorization scale relative to the Higgs mass, $\mu_{F} / m_{h}$, at the Tevatron and the LHC. The factorization scale is determined by the point at which the curves in Fig. 2 reach $85 \%$ of their values on the collinear plateau.

\begin{tabular}{lcc}
\hline \hline$m_{h}[\mathrm{GeV}]$ & Tevatron & LHC \\
\hline 100 & 0.203 & 0.227 \\
200 & 0.188 & 0.219 \\
300 & 0.176 & 0.215 \\
400 & 0.166 & 0.210 \\
500 & 0.157 & 0.206 \\
\hline \hline
\end{tabular}

the LHC, as would be expected if the collinear logarithms dominate. The situation at the Tevatron is more complicated. The ratio is near unity for Higgs masses of experimental interest, indicating that the calculation is reliable. However, as the Higgs mass increases the ratio grows, and continues to grow as the mass approaches the machine energy. This suggests that the calculation based on $b \bar{b} \rightarrow$ $h$ may be unreliable for very heavy Higgs bosons at the Tevatron.

In the full calculation of $b \bar{b} \rightarrow h$ (using the exact $b$ distribution function) it is important to choose the factorization scale near the end of the collinear plateau, but not very important exactly how that is defined. A less-than-

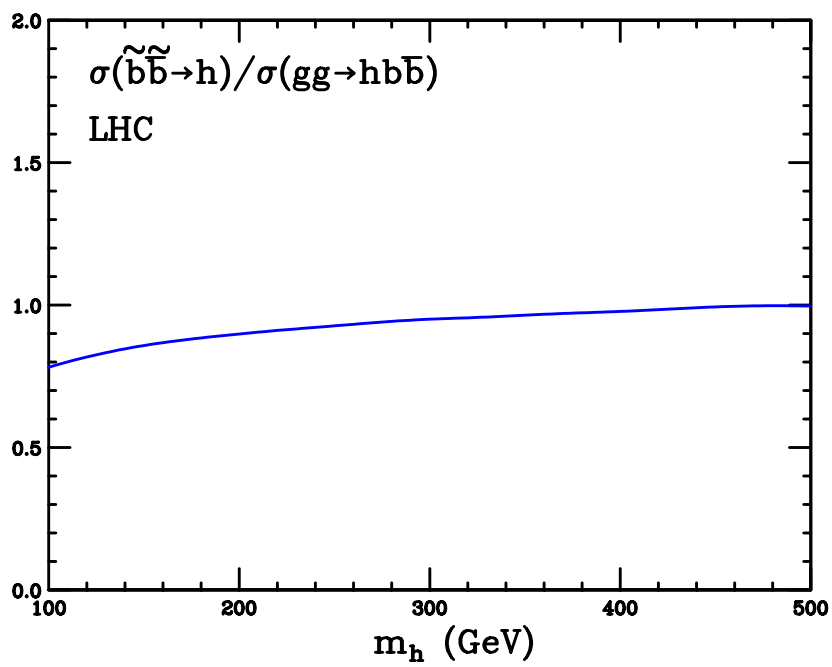

FIG. 3 (color online). $\quad \sigma(\tilde{b} \tilde{\tilde{b}} \rightarrow h) / \sigma(g g \rightarrow h b \bar{b})$ vs $m_{h}$ at the Tevatron and the LHC, using MRST2001 LO parton distribution functions [43], $m_{b}=4.7 \mathrm{GeV}$, and $\mu_{F}\left(=\mu_{R}\right)$ determined from the end of the collinear plateau in Fig. 2 (listed in Table I). 
optimal choice will be corrected by higher orders. Indeed, the next-to-next-to-leading-order (NNLO) calculation of $b \bar{b} \rightarrow h$ has very little factorization-scale dependence for values of $\mu_{F}$ near the end of the collinear plateau [21].

The advantage of the calculation based on the LO process $b \bar{b} \rightarrow h$ is actually twofold. As already discussed, it gives a more convergent perturbation series. In addition, it allows for a higher-order calculation than $g g \rightarrow h b \bar{b}$, since it is a simpler LO process. Indeed, $b \bar{b} \rightarrow h$ is known at NNLO [21], while $g g \rightarrow h b \bar{b}$ is known only at NLO [2224]. Thus the NNLO calculation of $b \bar{b} \rightarrow h$ is the most accurate existing calculation of inclusive Higgs-boson production in association with bottom quarks. This is reflected by the very mild dependence of the NNLO calculation of $b \bar{b} \rightarrow h$ on the factorization scale in comparison with that of the NLO calculation of $g g \rightarrow h b \bar{b}$ [25]. It would be interesting to study the behavior of the NNLO calculation for very heavy Higgs bosons at the Tevatron.

\section{III. $Z$ PRODUCTION IN ASSOCIATION WITH HEAVY QUARKS}

Unlike the case of the Higgs boson, there are a variety of contributions to the inclusive production of a $Z$ boson with heavy quarks. The analogue of the Higgs case is $b \bar{b} \rightarrow Z$, shown in Fig. 4. In the case of the $Z$ boson, one must also consider $c \bar{c} \rightarrow Z$ and $q \bar{q} \rightarrow Z(q=u, d, s)$, since both charm quarks and light quarks can fake a $b$ quark. Finally, there are the processes $q \bar{q} \rightarrow Z Q \bar{Q}$ and $q Q \rightarrow$ $Z q Q(Q=c, b)$, shown in Figs. 5 and 6 , where the $Z$ boson couples to the light quarks. As we will show, these last two processes are more important at the Tevatron than at the LHC.

Let us begin by considering only processes in which the $Z$ boson couples to the heavy quarks. This is completely analogous to the case of the Higgs boson discussed in the previous section. We will then include processes in which the $Z$ boson couples to light quarks, which have no analogue in the Higgs case.

The process $q \bar{q} \rightarrow Z$ has been calculated at NNLO [3638]. We modified this code to extract $Q \bar{Q} \rightarrow Z(Q=c, b)$ at NNLO, neglecting the heavy-quark mass, which is a small effect of order $\left(m_{Q} / M_{Z}\right)^{2} \times 1 / \ln ^{2}\left(M_{Z} / m_{Q}\right)$. We

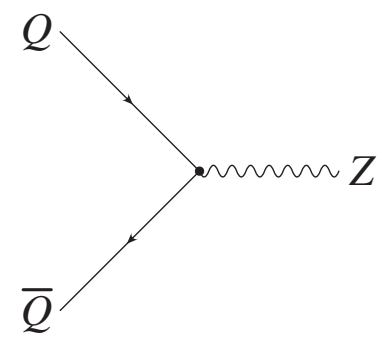

FIG. 4. Feynman diagram for $Q \bar{Q} \rightarrow Z(Q=c, b)$. The presence of heavy quarks in the final state is implied by the initialstate heavy quarks.

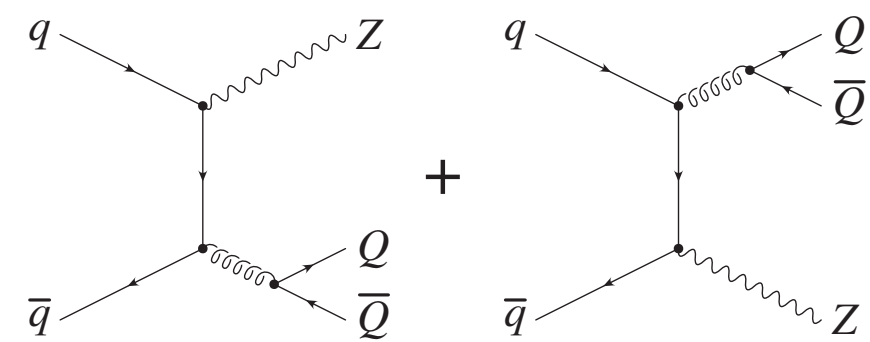

FIG. 5. Feynman diagrams for $q \bar{q} \rightarrow Z Q \bar{Q}$, where the $Z$ couples to the light quarks.

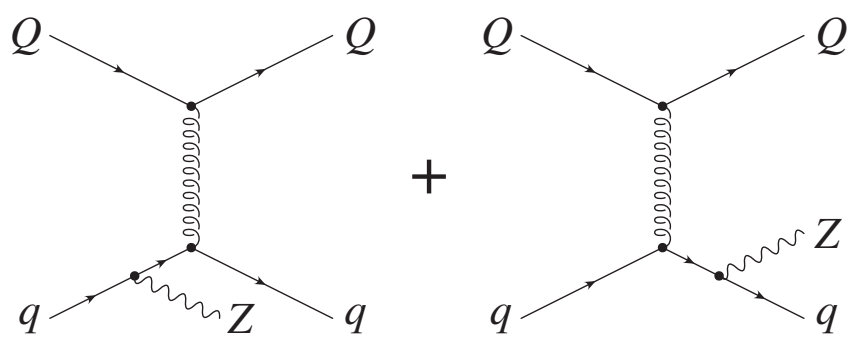

FIG. 6. Feynman diagrams for $q Q \rightarrow Z q Q$, where the $Z$ couples to the light quarks

keep (for the moment) only diagrams in which the $Z$ couples to the heavy quarks. ${ }^{4}$ We show in Fig. 7 the factorization-scale dependence of the cross section for $b \bar{b} \rightarrow Z$ at both the Tevatron and the LHC at LO, NLO, and NNLO. The renormalization scale has been set equal to the factorization scale, although this hardly matters as it first enters only at NLO, via the argument of $\alpha_{S}\left(\mu_{R}\right)$. As expected, the scale dependence decreases with increasing order, to the point where there is almost no scale dependence at NNLO. Similar results are obtained for $c \bar{c} \rightarrow Z$, as shown in Fig. 8.

Also shown on the same plot is the LO cross section in the four-flavor scheme, $g g \rightarrow Z b \bar{b}$. If we were to choose $\mu_{F}=\mu_{R}=M_{Z}$, this calculation would underestimate the true cross section by a factor of 4 at the Tevatron. If we choose the scale as in the previous section, by finding the end of the collinear plateau in $g b \rightarrow Z b$, we find that the appropriate factorization scale is around $M_{Z} / 3$ at both the Tevatron and the LHC. With this choice of scale, the factor of 4 is reduced to 2 . For charm, the corresponding factor is 5 at the Tevatron, reduced to 3 . These results mirror a similar result that was obtained in the case of the Higgs [20].

\footnotetext{
${ }^{4}$ This includes NNLO processes with four external heavy quarks of the same flavor. However, we do not include processes with two external charm and two external bottom quarks. These processes contribute less than $1 \%$ of the LO cross section.
} 

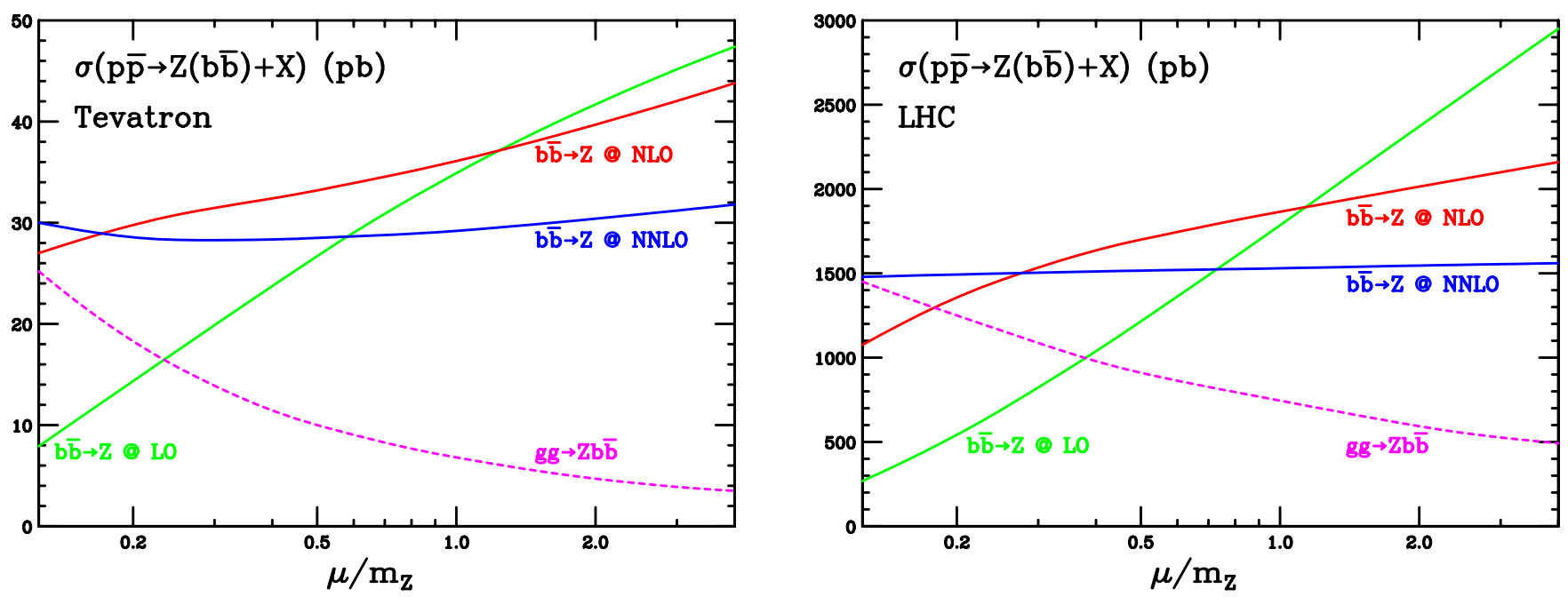

FIG. 7 (color online). Factorization-scale dependence of $b \bar{b} \rightarrow Z$ at LO, NLO, and NNLO at the Tevatron and the LHC. Only processes in which the $Z$ couples to the heavy quarks are included. Also shown is $g g \rightarrow Z b \bar{b}$ at LO, using $m_{b}=4.7 \mathrm{GeV}$. We use the LO, NLO, and NNLO parton distribution functions MRST2001 LO [43] and MRST2002 [35].
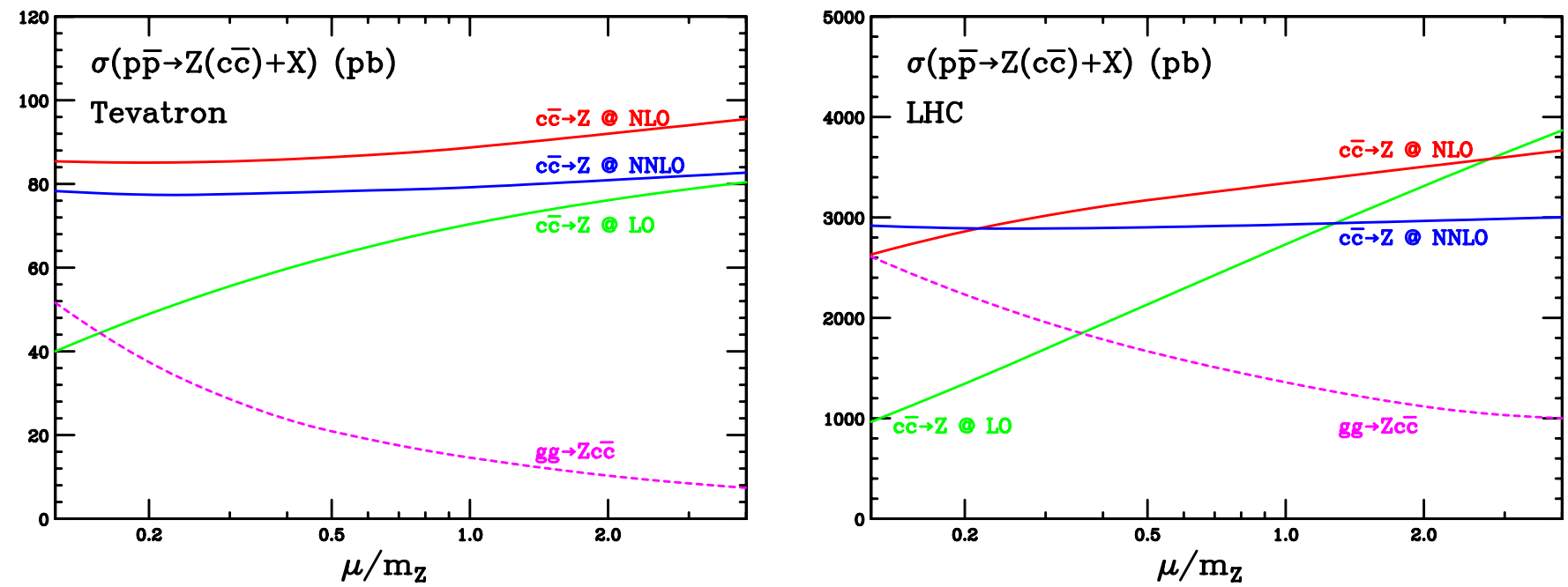

FIG. 8 (color online). $\quad$ Same as Fig. 7, but for $c \bar{c} \rightarrow Z$. For $g g \rightarrow Z c \bar{c}$, we use $m_{c}=1.4 \mathrm{GeV}$.

We list in Table II the NNLO cross sections for $b \bar{b} \rightarrow Z$ and $c \bar{c} \rightarrow Z$ at both the Tevatron and the LHC. These cross sections have very little theoretical uncertainty.

We now include processes in which the $Z$ couples to light quarks, shown in Figs. 5 and $6 .{ }^{5}$ Here it is essential to keep the heavy-quark mass nonzero in order to regulate collinear singularities. ${ }^{6}$ While these processes are NNLO

\footnotetext{
${ }^{5}$ We also consider the process $Q \bar{Q} \rightarrow Z q \bar{q}$, where the $Z$ couples to the light quarks. We find this to be numerically negligible. The interference with the same process, but where the $Z$ couples to the heavy quarks, is also negligible.

${ }^{6} \mathrm{We}$ also evaluate the interference of these processes with the similar processes in which the $Z$ is radiated from the heavy quark. This may be done in the limit of vanishing heavy-quark mass, since there are no collinear singularities. We find that these interference contributions are numerically negligible.
}

with respect to inclusive $Z$ production, $q \bar{q} \rightarrow Z Q \bar{Q}$ is $\mathrm{LO}$ with respect to $Z$ production in association with heavy quarks, and $q Q \rightarrow Z q Q$ is NLO. The correct power counting is obtained when one recalls that a heavy-quark distribution function is intrinsically of order $\alpha_{S} \ln \left(\mu_{F} / m_{Q}\right)$ [39]. The analogous processes for heavy-quark structure functions in deep-inelastic scattering, $F_{i}^{Q}$, have been discussed in Ref. [40].

There are two serious drawbacks to the calculations of these processes. First, the cross sections contain factors of $\ln \left(M_{Z} / m_{Q}\right)$, due to the collinear singularities, which are not resummed. This is related to the fact that we are calculating a semi-inclusive quantity, namely $Z$ production in association with heavy quarks. If we were instead calculating the inclusive $Z$ cross section, this issue would not arise. Fracture functions may be useful in this context [41]. 
TABLE II. Cross sections $(\mathrm{pb})$ for the various contributions to $Z$ production in association with heavy quarks. We use the MRST2002 NNLO parton distribution functions [35] with $\mu_{F}=$ $\mu_{R}=M_{Z} / 3$.

\begin{tabular}{lccc}
\hline \hline Process & & Tevatron & LHC \\
\hline$Z(b \bar{b})$ & $b \bar{b} \rightarrow Z(\mathrm{NNLO})$ & 28.3 & 1500 \\
& $q \bar{q} \rightarrow Z b \bar{b}(\mathrm{LO})$ & 19 & 120 \\
& $q b \rightarrow Z q b(\mathrm{LO})$ & 5.9 & 430 \\
$Z(c \bar{c})$ & $c \bar{c} \rightarrow Z$ (NNLO) & 77.7 & 2890 \\
& $q \bar{q} \rightarrow Z c \bar{c}(\mathrm{LO})$ & 69 & 430 \\
& $q c \rightarrow Z q c(\mathrm{LO})$ & 21 & 1200 \\
Inclusive $Z$ & & 7510 & 56700 \\
\hline \hline
\end{tabular}

Second, and more importantly, these processes are only known at LO at this time (with a nonzero quark mass). ${ }^{7}$ The NLO calculation is an important missing result for this as well as many other analyses (the same holds true of $q \bar{q} \rightarrow W Q \bar{Q})$. Thus our calculation of these processes is relatively crude. This is a serious problem at the Tevatron, but less so at the LHC, where these processes are relatively less important. It is desirable both to obtain NLO results for $q \bar{q} \rightarrow Z Q \bar{Q}$ and $q Q \rightarrow Z q Q$ (with finite $m_{Q}$ ) and to develop a formalism that allows the resummation of the collinear logarithms. ${ }^{8}$

We give in Table II the cross sections for the various processes that contribute to $Z$ production in association with heavy quarks. We also give the inclusive $Z$ cross section at NNLO. Although this cross section is 2 orders of magnitude larger than that of any of the processes that

\footnotetext{
${ }^{7}$ The process $q \bar{q} \rightarrow Z Q \bar{Q}$ is known at NLO with a vanishing quark mass, which is relevant when the heavy quarks are produced at high $p_{T}$ [42].

${ }^{8}$ The collinear logarithm that occurs in $q Q \rightarrow Z q Q$ also occurs in the process $q g \rightarrow Z q Q \bar{Q}$, where the initial gluon splits to $Q \bar{Q}$. Thus the same issues arise in a calculation that does not make use of a heavy-quark distribution function.
}

produce a $Z$ in association with heavy quarks, the mistag rate for light quarks and gluons is on the order of $1 \%$, so this background is not overwhelming.

\section{CONCLUSIONS}

Our results for $Z$ production in association with heavy quarks are summarized in Table II. The final row is the inclusive $Z$ cross section calculated at NNLO. Each row above that corresponds to some subset of this calculation, with heavy quarks in the final state, either implicitly (such as $Q \bar{Q} \rightarrow Z$ ) or explicitly (such as $q \bar{q} \rightarrow Z Q \bar{Q}$ ). We see that there are a large variety of processes that contribute to $Z$ production in association with heavy quarks. Taken together, they constitute $3 \%$ of the inclusive $Z$ cross section at the Tevatron, and $12 \%$ at the LHC. The measurement of these fractions will require simulation of the acceptances and tagging efficiencies of the various processes. We advocate using an inclusive tagging technique to maximize the number of signal events.

The measurement of $Z$ production in association with heavy quarks is interesting in its own right, but also as a feasibility study for Higgs production in association with bottom quarks. In this paper we have argued that the best formalism for the calculation of the latter process is based on the leading-order process $b \bar{b} \rightarrow h$, and that this calculation is valid for all Higgs masses of interest at both the Tevatron and the LHC. The most accurate calculation of this process is the NNLO cross section given in Ref. [21]. We also showed evidence that this formalism fails as the Higgs mass approaches the machine energy.

\section{ACKNOWLEDGMENTS}

We are grateful for conversations about theoretical issues with J. Collins, S. Forte, and F. Olness, and about experimental issues with T. Liss, S. Marcellini, G. Masetti, S. Nikitenko, and K. Pitts. This work was supported in part by the U.S. Department of Energy under Contract No. DEFG02-91ER40677.
[1] D. A. Dicus and S. Willenbrock, Phys. Rev. D 39, 751 (1989).

[2] Z. Kunszt and F. Zwirner, Nucl. Phys. B385, 3 (1992).

[3] C. Kao and N. Stepanov, Phys. Rev. D 52, 5025 (1995).

[4] J. Dai, J. F. Gunion, and R. Vega, Phys. Lett. B 345, 29 (1995).

[5] J. Dai, J. F. Gunion, and R. Vega, Phys. Lett. B 387, 801 (1996).

[6] E. Richter-Was and D. Froidevaux, Z. Phys. C 76, 665 (1997).

[7] V. D. Barger and C. Kao, Phys. Lett. B 424, 69 (1998).
[8] D. Choudhury, A. Datta, and S. Raychaudhuri, hep-ph/ 9809552

[9] C.S. Huang and S.H. Zhu, Phys. Rev. D 60, 075012 (1999).

[10] M. Drees, M. Guchait, and P. Roy, Phys. Rev. Lett. 80, 2047 (1998); 81, 2394(E) (1998).

[11] M. Carena, S. Mrenna, and C. E. Wagner, Phys. Rev. D 60, 075010 (1999).

[12] J. L. Diaz-Cruz, H. J. He, T. Tait, and C.P. Yuan, Phys. Rev. Lett. 80, 4641 (1998).

[13] C. Balazs, J.L. Diaz-Cruz, H. J. He, T. Tait, and C.P. Yuan, Phys. Rev. D 59, 055016 (1999). 
[14] C. Balazs, H. J. He, and C.P. Yuan, Phys. Rev. D 60, 114001 (1999).

[15] D. Dicus, T. Stelzer, Z. Sullivan, and S. Willenbrock, Phys. Rev. D 59, 094016 (1999).

[16] ATLAS Collaboration, Technical Design Report No. CERN-LHCC-99-15.

[17] M. Carena et al., hep-ph/0010338.

[18] T. Affolder et al. (CDF Collaboration), Phys. Rev. Lett. 86, 4472 (2001).

[19] J. Campbell, R. K. Ellis, F. Maltoni, and S. Willenbrock, Phys. Rev. D 67, 095002 (2003).

[20] F. Maltoni, Z. Sullivan, and S. Willenbrock, Phys. Rev. D 67, 093005 (2003).

[21] R. V. Harlander and W. B. Kilgore, Phys. Rev. D 68, 013001 (2003).

[22] S. Dittmaier, M. Kramer, and M. Spira, Phys. Rev. D 70, 074010 (2004).

[23] S. Dawson, C. B. Jackson, L. Reina, and D. Wackeroth, Phys. Rev. D 69, 074027 (2004).

[24] S. Dawson, C. B. Jackson, L. Reina, and D. Wackeroth, Phys. Rev. Lett. 94, 031802 (2005).

[25] J. Campbell et al., hep-ph/0405302.

[26] V. Abazov (D0 Collaboration), hep-ex/0504018.

[27] D. Acosta et al. (CDF Collaboration), Phys. Rev. D 71, 092001 (2005).

[28] J. Campbell, R. K. Ellis, F. Maltoni, and S. Willenbrock, Phys. Rev. D 69, 074021 (2004).
[29] V. M. Abazov et al. (D0 Collaboration), Phys. Rev. Lett. 94, 161801 (2005).

[30] M. A. Aivazis, J. C. Collins, F. I. Olness, and W. K. Tung, Phys. Rev. D 50, 3102 (1994).

[31] J. C. Collins, Phys. Rev. D 58, 094002 (1998).

[32] M. Krämer, Nucl. Phys. B Proc. Suppl. 135, 66 (2004).

[33] T. Plehn, Phys. Rev. D 67, 014018 (2003).

[34] E. Boos and T. Plehn, Phys. Rev. D 69, 094005 (2004).

[35] A. D. Martin, R. G. Roberts, W. J. Stirling, and R. S. Thorne, Phys. Lett. B 531, 216 (2002).

[36] R. Hamberg, W. L. van Neerven, and T. Matsuura, Nucl. Phys. B359, 343 (1991); B644, 403(E) (2002).

[37] P. J. Rijken and W. L. van Neerven, Phys. Rev. D 52, 149 (1995).

[38] R. V. Harlander and W. B. Kilgore, Phys. Rev. Lett. 88, 201801 (2002).

[39] T. Stelzer, Z. Sullivan, and S. Willenbrock, Phys. Rev. D 56, 5919 (1997).

[40] A. Chuvakin, J. Smith, and W. L. van Neerven, Phys. Rev. D 61, 096004 (2000).

[41] L. Trentadue and G. Veneziano, Phys. Lett. B 323, 201 (1994).

[42] J. M. Campbell and R. K. Ellis, Phys. Rev. D 62, 114012 (2000).

[43] A. D. Martin, R. G. Roberts, W. J. Stirling, and R. S. Thorne, Eur. Phys. J. C 23, 73 (2002). 